\title{
FORMULATION AND CHARACTERIZATION OF HPMC AND HPMCAS BASED SOLID DISPERSIONS OF FENOFIBRATE: A COMPARATIVE STUDY
}

\author{
ANKIT RAMPAL, MANMEET SINGH, SHANTA MAHAJAN, NEENA BEDI*
}

Department of Pharmaceutical Sciences, Guru Nanak Dev University, Amritsar, Punjab, India (143005)

Email: neena.pharma@gndu.ac.in

Received: 16 Feb 2019, Revised and Accepted: 11 Apr 2019

\begin{abstract}
Objective: The aim of the present study was to investigate the effect of novel polymeric carriers and to develop solid dispersion formulation that could improve in vitro profile of Fenofibrate (FB).

Methods: Spray drying technique was used to fabricate solid dispersions with hydrophilic carriers, mainly hydroxypropyl methylcellulose (HPMC) and hydroxypropyl methylcellulose acetate succinate (HPMCAS). Solid dispersions in the form of spray-dried powder were characterized with respect to the pure drug and the corresponding physical mixtures by optical microscopy, x-ray diffraction (XRD), fourier transform infrared (FT-IR) spectroscopy and differential scanning calorimetry (DSC). Size and morphology of optimized solid dispersion were performed by scanning electron microscopy (SEM). Furthermore, in vitro dissolution comparisons were carried out between the optimized solid dispersion against the pure drug and the physical mixtures.

Results: Solubility studies demonstrated that the solubility of FB was not affected by pH change. The transformation of crystalline FB into an amorphous solid dispersion powder has been clearly demonstrated by optical microscopy. The molecular dispersion of drug in the dispersion matrix prepared by spray drying was confirmed in XRD and DSC studies. IR spectroscopy was observed with negligible incompatibility of the drug with polymers. Spherical morphology was observed in SEM with no evidence of FB crystals. The prepared solid dispersions exhibited dissolution improvement as compared to the pure drug and spray dried FB in 0.05 M SLS, with HPMCAS as the superior carrier over HPMC.
\end{abstract}

Conclusion: The present study vouches better in vitro profile of FB from spray-dried HPMCAS based solid dispersions.

Keywords: Solubility, HPMC, HPMCAS, Solid dispersions, Crystallinity

(C) 2019 The Authors. Published by Innovare Academic Sciences Pvt Ltd. This is an open-access article under the CC BY license (http://creativecommons.org/licenses/by/4.0/) DOI: http://dx.doi.org/10.22159/ijap.2019v11i4.32592

\section{INTRODUCTION}

About $40 \%$ of New Chemical Entities in drug development pipelines suffered from poor water solubility or dissolution rate-limited absorption which eventually falls into the Biopharmaceutics Classification System (BCS) Class II and thus they fail to reach the market. These poorly water-soluble drugs are associated with slow drug absorption leading to inadequate and variable bioavailability and gastrointestinal mucosal toxicity [1]. Techniques have been developed to address the low aqueous solubility challenges, including chemical modification, such as pro-drugs and salt formation, or formulation methods such as particle size reduction, co-crystal formation, inclusion complexes using cyclodextrins and lipid formulations and solid form changes such as nanocrystals and amorphous dispersions of API and polymers [2]. Of these techniques, amorphous solid dispersion is a useful approach to increase the dissolution rate of poorly water-soluble drugs and thereby improve their bioavailability, although this must be proved for each drug. The preparation of the solid dispersion involves drug deposition on the surface of an inert carrier which results in a greater surface area of the drug leading to a faster rate of dissolution [3]. Recently used methods for the preparation of amorphous solid dispersions include mechanical grinding, melting, hot melt extrusion, spray drying, lyophilization and supercritical fluid precipitation [4]. Spray drying is one of the effective methods for preparing amorphous solid dispersion which consists of suspending the drug and the polymer in a common solvent and then drying it to form uniform nanoparticulate size powder [5]. The scalability and efficient critical process parameters of spray drying technology based solid dispersions are widely applied in the pharmaceutical industry, which provides equalized content uniformity and nanosized distributed solid surfaces.

FB, a prodrug of fenofibric acid, is used for the treatment of hypertriglyceridemia, mixed dyslipidemia, and hypercholesterolemia, as it can reduce levels of triglycerides, total cholesterol and low-density lipoprotein [6]. However, FB is a neutral lipophilic drug ( $\log \mathrm{P}=5.2$ ), which is practically insoluble in water. It is classified as a class II BCS drug and oral bioavailability of approximately $30 \%$ is reported in humans. Many nanoformulations like mesoporous solid particles, liposomes or tablet approaches have been studied to improve the solubility of FB. However, the majority of these formulations used either a special matrix of mesoporous surfaces, superdisintegrants or surfactants as modified excipients lacking long-term biocompatibility or involved technically challenging processes. Some reports have confirmed that silica can act as an immunogenic sensitizer and induce contact hypersensitivity. Furthermore, the formulation must be carefully designed because the pore architecture of silica may greatly influence its biocompatibility, and high dose and long-term usage should be avoided $[5,6]$. The spray drying technique used for the preparation of the solid dispersion using different hydrophilic polymers such as HPMC and HPMCAS has not been explored to improve the aqueous solubility of FB. In the present study, an attempt was made to develop and optimize a solid dispersion system using different proportions of drug per carrier. Furthermore, the present study aims to clarify the potential for improving the solubility and dissolution rate of FB using hydrophilic polymers such as HPMC and HPMCAS by spray drying technology.

\section{MATERIALS AND METHODS}

HPMCAS-LF and HPMC (K100 Grade) were obtained from signet chemicals corporation, Mumbai. FB was procured from macleod's pharmaceuticals, Mumbai. HPLC grade acetonitrile, hydrochloric acid $(\mathrm{HCl})$, and dichloromethane were purchased from SD fine chemicals llimited, India. Methanol and potassium dihydrogen phosphate were purchased from rankem India. Phosphoric acid was purchased from fischer scientific limited, India. All chemicals and reagents utilized were of analytical grade. Triple distilled water (Rions, India) was used throughout the entire study.

High-performance liquid chromatography (HPLC) analysis of FB

Reversed-phase column based high-performance liquid chromatographic method (Nexera X2, Shimadzu, Japan) was used for quantification of FB in analytical media. The LC system consisted of a 
pump configured to Lab Solutions software with auto-injecting facility. The HPLC was equipped with a column oven and the analytical column used was Enable C-18; $4.6 \mathrm{~mm} \times 250 \mathrm{~mm} ; 5 \mu \mathrm{m}$ in diameter with an injection loop of $20 \mu$ l. The mobile phase used was acetonitrile and acidified water $\mathrm{pH}$ of water adjusted with phosphoric acid to $2.5 \pm 0.1)(70: 30)$ at a flow rate of $1 \mathrm{ml} / \mathrm{min}$. The injection volume was $5 \mu \mathrm{l}$ with a run time of $20 \mathrm{~min}$ and the drug peak was observed at $286 \mathrm{~nm}$ through photodiode array (PDA) detector. Column equilibration was done at least $30 \mathrm{~min}$ prior to the injection of the drug solution [7].

\section{Solubility determination in different media}

Equilibrium solubility of $\mathrm{FB}$ was determined at $37 \pm 1{ }^{\circ} \mathrm{C}$ under physiological $\mathrm{pH}$ conditions i.e. $0.1 \mathrm{~N} \mathrm{HCl}$, phosphate buffer (PB) $\mathrm{pH}$ 4.5, pH 6.8 and in different media such as distilled water and sodium lauryl sulphate (SLS) $25 \mathrm{mmol}$ and $0.05 \mathrm{M}$ using validated shake flask method [8]. An excess amount of drug i.e. $10 \mathrm{mg}$ of FB was added to glass vials containing $5 \mathrm{ml}$ of each medium and allowed to equilibrate for $24 \mathrm{~h}$. The content of each vial was filtered through a $0.22 \mu \mathrm{m}$ membrane filter (Millipore, India) and was analyzed using HPLC.

\section{Preparation of binary systems \\ Preparation of HPMC and HPMCAS physical mixtures of FB}

For the preparation of physical mixtures, FB and polymers were added in different ratios $(2: 1,1: 1$ and $1: 2)$ and were mixed thoroughly with the help of pestle-mortar to obtain homogeneous mixtures (table 1). The resulting mixture was passed through sieve $\# 40$ and was stored in a desiccator until use [9].

Preparation of spray dried FB and solid dispersions of FB with HPMC and HPMCAS

Spray dried FB was prepared to check if the process i.e. spray drying has any effect on the crystallinity of pure drug. FB was dissolved in methanol to form a $2 \% w / v$ solution. This solution was sonicated for $5 \mathrm{~min}$. to obtain a clear solution. It was subjected to spray drying (Lab Ultima, LU 228 Advanced, Mumbai), keeping the inlet temperature at $50{ }^{\circ} \mathrm{C}$; outlet temperature $40^{\circ} \mathrm{C}$; feed pump flow rate $3 \mathrm{ml} / \mathrm{min}$; aspirator speed $65 \mathrm{~m}^{3} / \mathrm{h}$, a vacuum of $90 \mathrm{~mm} / \mathrm{Hg}$ and atomization pressure at $1.2 \mathrm{~kg} / \mathrm{cm}^{2}$. Solid dispersions of FB were also prepared in the following compositions by spray drying using the above-mentioned conditions (table 1) [9].

Table 1: Physical mixtures and spray dried solid dispersions composition

\begin{tabular}{llll}
\hline & & Spray dried solid dispersions composition & Ratio \\
\hline FB: HPMC & Ratio & FB: HPMC & $2: 1$ \\
PMC1 & $2: 1$ & SD MC1 & $1: 1$ \\
PMC2 & $1: 1$ & SD MC2 & $1: 2$ \\
PMC3 & $1: 2$ & SD MC3 & $2: 1$ \\
FB: HPMCAS & & FB: HPMCAS & $1: 1$ \\
PM CAS4 & $2: 1$ & SD CAS4 & $1: 2$ \\
PM CAS5 & $1: 1$ & SD CAS5 & \\
PM CAS6 & $1: 2$ & SD CAS6 & \\
\hline
\end{tabular}

\section{Characterizations of physical mixtures and solid dispersions \\ Optical and polarized microscopy}

Each sample ( $<0.1 \mathrm{mg}$ ) was mounted on a glass slide with a brush, covered with silicone oil and a coverslip and was observed under the microscope (Lecia DMLP, Germany). Light intensity was adjusted and observations were done under normal and polarized light (by engaging the polarizer). Birefringence patterns were observed at a high-resolution scale and images were acquired.

\section{FT-IR spectroscopic analysis}

FT-IR Spectrograms were obtained using FT-IR spectrometer (Perkin Elmer, USA) by the conventional $\mathrm{KBr}$ pellet method [9]. The samples were grounded gently with anhydrous $\mathrm{KBr}$ and compressed to form pellets and the scanning was carried out in a range of $4000-400 \mathrm{~cm}^{-1}$.

\section{DSC analysis}

Each sample ( $2 \mathrm{mg}$ ) was scanned in an aluminum pan over the range of $20{ }^{\circ} \mathrm{C}$ to $220{ }^{\circ} \mathrm{C}$, at a rate of $10{ }^{\circ} \mathrm{C} / \mathrm{min}$ under the controlled environment of liquid nitrogen at a rate of $50 \mathrm{ml} / \mathrm{min}$. Thermal data analysis of the DSC thermograms was conducted using TA Universal Analysis (DSC Q 20-2661 TA Instruments, USA).

\section{XRD analysis}

XRD measurements were performed using X-ray diffractometer (X'PERT PRO, PANalytical, Netherlands). Samples were irradiated with monochromatic $\mathrm{Cu}$ radiation and patterns were obtained by continuous scanning at a step size of $0.017^{\circ}$, with detector resolution in $2 \theta$ (diffraction angle) between $3.5^{\circ}$ to $50 \theta$. The generator settings used were $40 \mathrm{~mA}$ and intensity resolution of $45 \mathrm{kV}$ at ambient temperature [10].

\section{Scanning electron microscopy}

The electron microscopy analysis was carried out to study the surface morphology of optimized dispersions in a scanning electron microscope (Carl Zeiss SUPRA 55). Samples were gold coated and mounted on the stubs using double-sided carbon adhesive tape and were analyzed in different magnification scales at $100 \mathrm{kV}$ ionizing radiations [11].

\section{In vitro dissolution studies}

FB, spray dried FB, physical mixtures and solid dispersions equivalent to $54 \mathrm{mg}$ of drug were subjected to in vitro dissolution testing using USP Type II (Paddle type) Dissolution Apparatus (Electrolab TDT-08l Dissolution Tester, India), using $900 \mathrm{ml}$ of $0.05 \mathrm{M}$ SLS as dissolution medium. The rotation speed of paddle was kept at $50 \mathrm{rpm}$ and the temperature was set at $37 \pm 2{ }^{\circ} \mathrm{C}$. Samples $(5$ $\mathrm{ml}$ ) were withdrawn at intervals of 5, 10, 15, 30, 45, 60, 90, $120 \mathrm{~min}$. and were replaced with an equal amount of dissolution medium to reimburse the loss during sampling After each withdrawal, aliquots were filtered through a $0.2 \mu \mathrm{m}$ membrane filter (Millipore, India) and analyzed using optimized HPLC method $[10,11]$.

\section{Calculation of dissolution parameters}

Percent Dissolution Efficiency (DE) for each formulation was computed as the percent ratio of an area under the dissolution curve up to the time, $t$, to that of the area of the rectangle described by $100 \%$ dissolution at the same time and was calculated by using equation 1

$$
D E=\left(\frac{\int_{0}^{t} y \cdot d t}{y 100 . t}\right) 100----------1
$$

Where $\mathrm{y}$ is the percent drug dissolved at time $\mathrm{t}$. For these formulations, $\mathrm{DE}_{30}$ and $\mathrm{DE}_{60}$ were calculated. Other dissolution parameters such as $t_{60}, \mathrm{DP}_{5}, \mathrm{DP}_{30}$, and $\mathrm{DP}_{120}$ were also calculated $\left(t_{60}\right.$ is the time taken to release $60 \%$ of the drug and DP is the percent drug released at a particular time).

\section{RESULTS AND DISCUSSION}

\section{Solubility determination in different media}

Results from saturation solubility studies demonstrated that there was no significant difference between saturation solubility values of 
FB at different $\mathrm{pH}$. This is attributed to the fact that it has no ionizable group, so its solubility is not influenced by a change in $\mathrm{pH}$. The solubility of FB increased significantly in $25 \mathrm{mmol}$ SLS and 0.05
M SLS solutions which might be attributed to its micellar solubilization property [12]. The solubility data in various media are given in fig. 1.

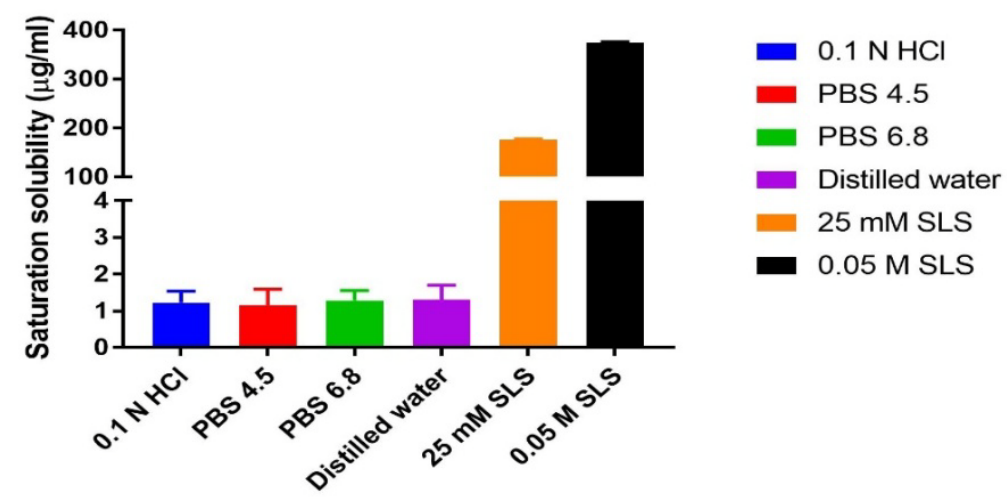

Fig. 1: Saturation solubility of FB in different media (mean $\pm S D, n=3$ )

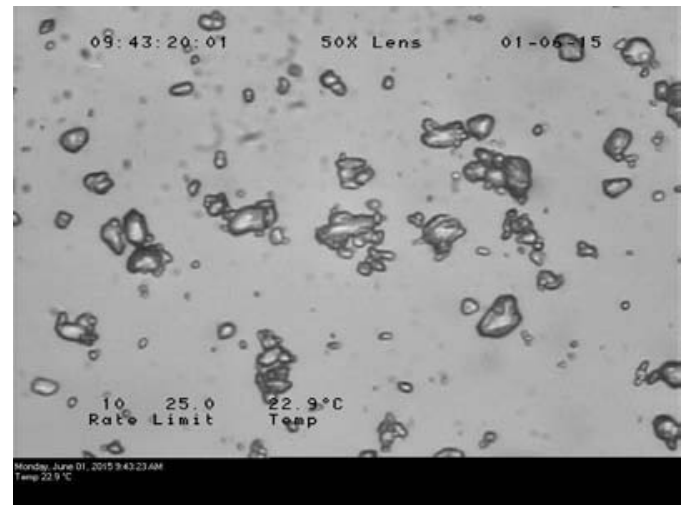

(a1)

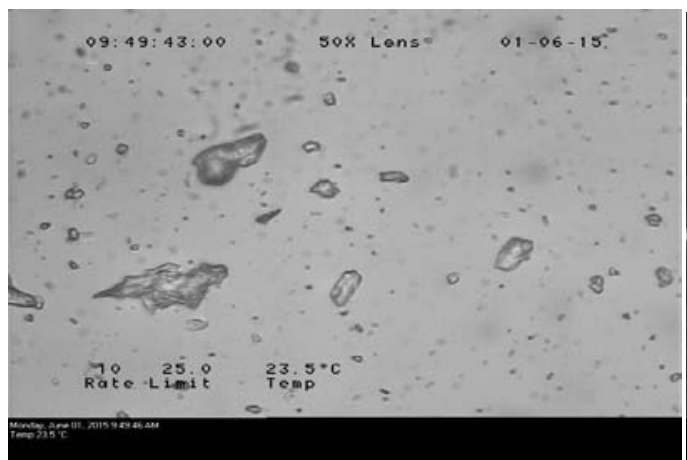

(b1)

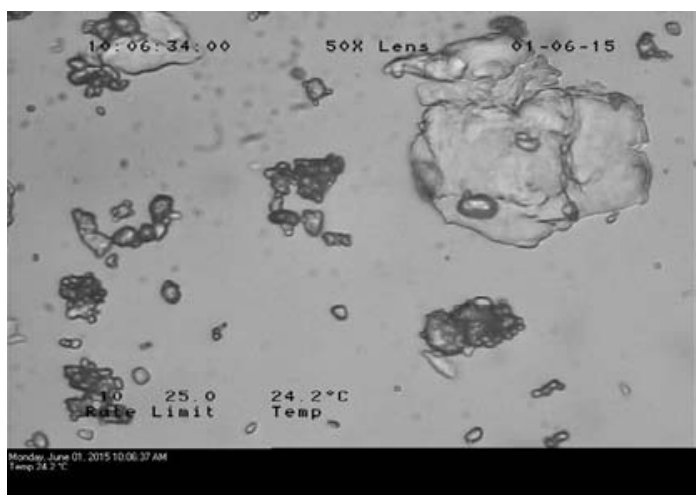

(c1)

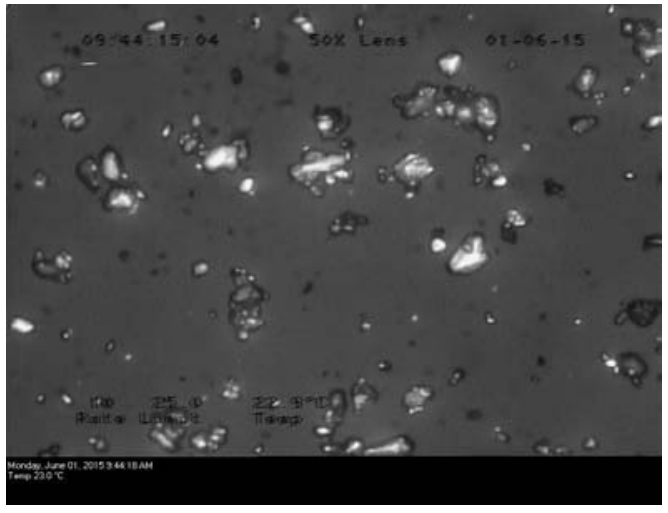

(a2)

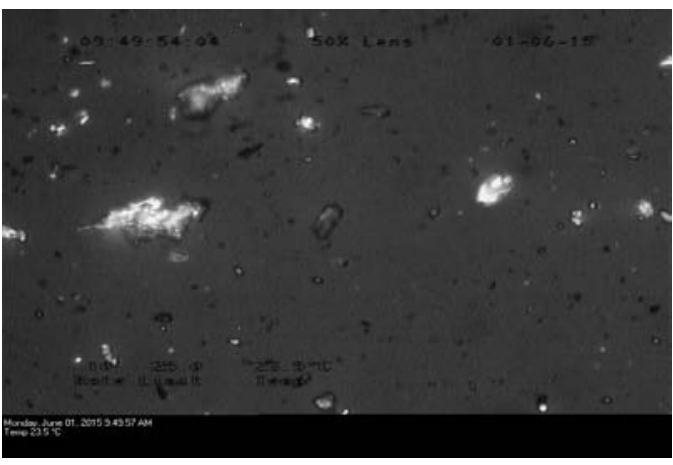

(b2)

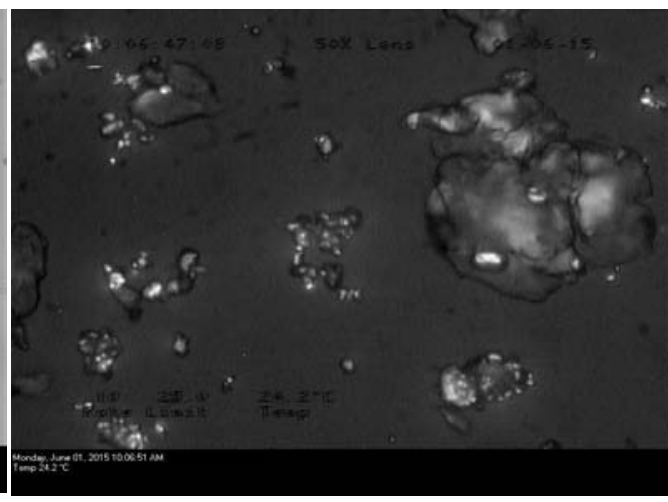

(c2) 


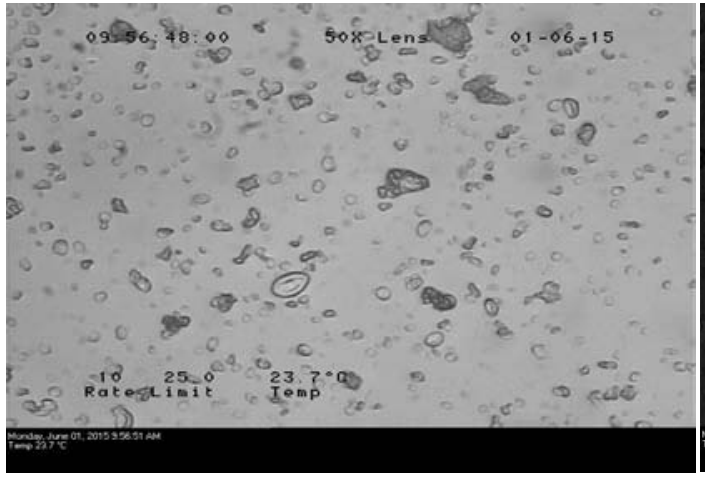

(d1)

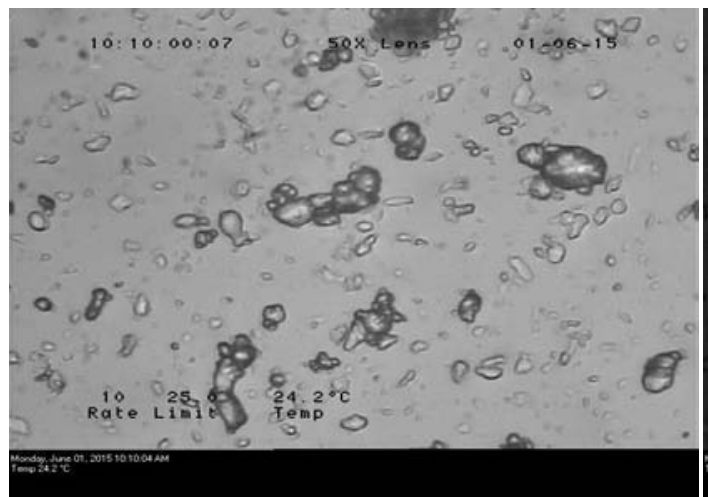

(e1)

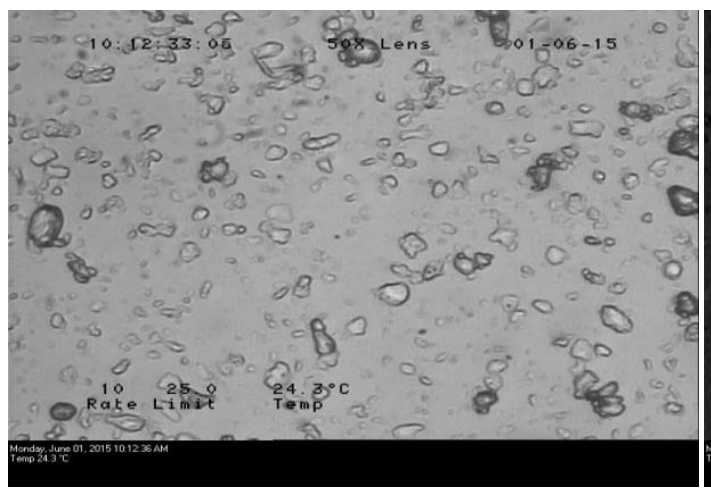

(f1)

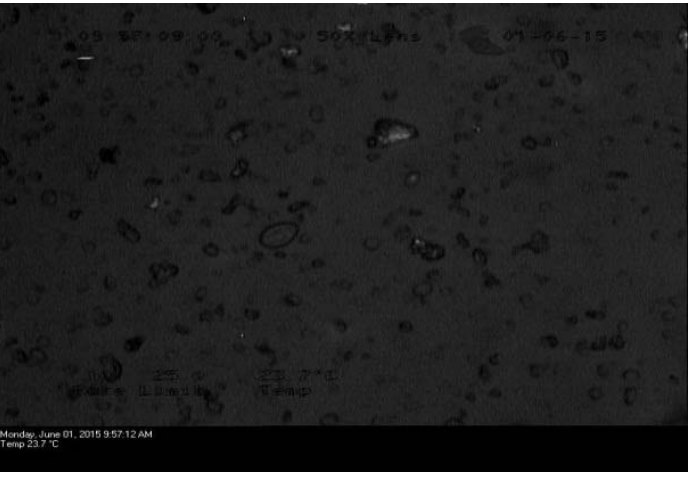

(d2)

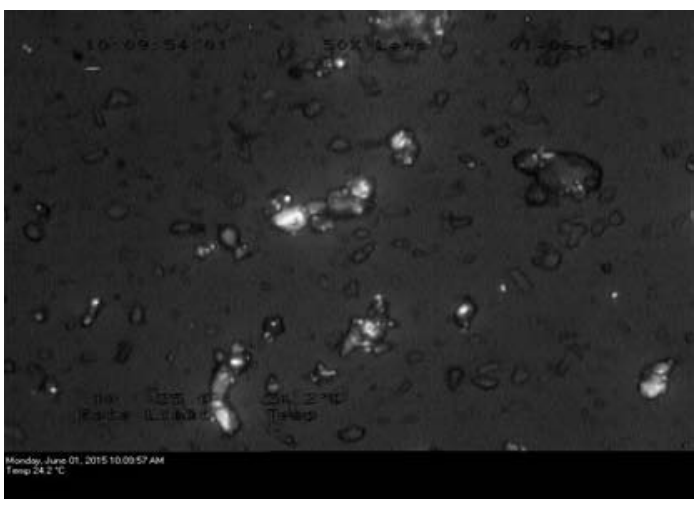

(e2)

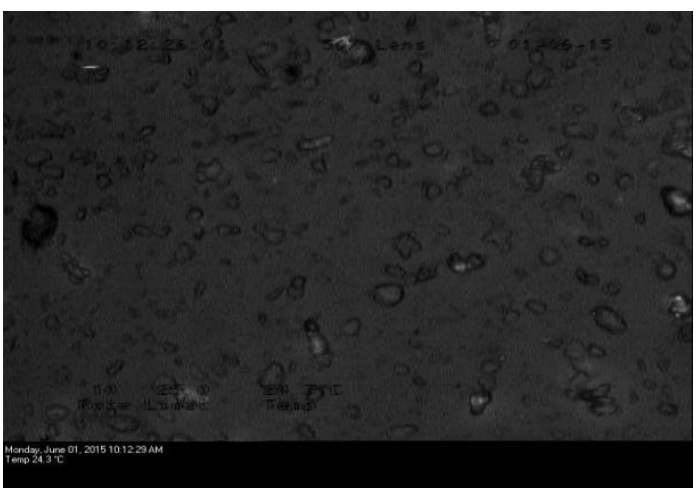

(f2)

Fig. 2: Optical micrographs of (a1) FB (b1) Spray-dried FB (c1) HPMC physical mixture (d1) HPMC solid dispersion (e1) HPMCAS physical mixture (f1) HPMCAS solid dispersion. Polarized micrographs of (a2) FB (b2) Spray-dried FB (c2) HPMC physical mixture (d2) HPMC solid dispersion (e2) HPMCAS physical mixture (f2) HPMCAS solid dispersion

Characterizations of FB, spray dried FB, HPMC, HPMCAS, physical mixtures and solid dispersions

\section{Optical and polarized microscopy}

Optical and polarized microscopy was done to observe the birefringence pattern. Fig. 2 showed optical and polarized micrographs of the drug, polymeric carriers, physical mixtures and solid dispersions. Presence of birefringence confirms the presence of crystallinity in the samples. However, loss of crystallinity was observed in prepared physical mixtures and solid dispersions by uniform dispersion of the drug in molecular matrix. This phenomenon suggested effective encapsulation of FB into the hydrophilic matrix of HPMC and HPMCAS with no evidence of FB crystals on the surface.

\section{FT-IR spectroscopic analysis}

The IR spectrum provides information about the chemical bonds, characteristics functional group and usually detects interactions between drug and carrier in the solid dispersion. The spectrum overlay plot of the drug, polymers, physical mixtures and optimized solid dispersions are depicted in fig. 3. The IR spectrum of the drug showed major peaks at various C-H stretches from $2872 \mathrm{~cm}^{-1}$ to $3068 \mathrm{~cm}^{-1}$. The $\mathrm{C}=\mathrm{C}$ stretches were observed at $1594 \mathrm{~cm}^{-1}$ and the bands at $1173 \mathrm{~cm}^{-1}$ and $1291 \mathrm{~cm}^{-1}$ were attributed to C-O stretches. The spectra for the raw and spray-dried drug were similar, confirming that the drug did not undergo any transformation after spray drying [5]. The IR spectra of the solid dispersion and the physical mixture of the drug with HPMC and HPMCAS clearly showed that there is no incompatibility of the drug with the polymer. No significant shifts were observed in the IR spectrum of the solid dispersion and all the stretching as well as bending vibrations such as $\mathrm{OH}$ stretch, $\mathrm{C}-\mathrm{H}$ stretches, C-O stretch, $\mathrm{C}=\mathrm{C}$ stretch, $\mathrm{C}-\mathrm{H}$ bending, $\mathrm{C}-\mathrm{Cl}$ stretches were observed. These findings observed negligible chemical interaction between drug and polymers and successfully be transformed into an effective pharmaceutical dosage form. 


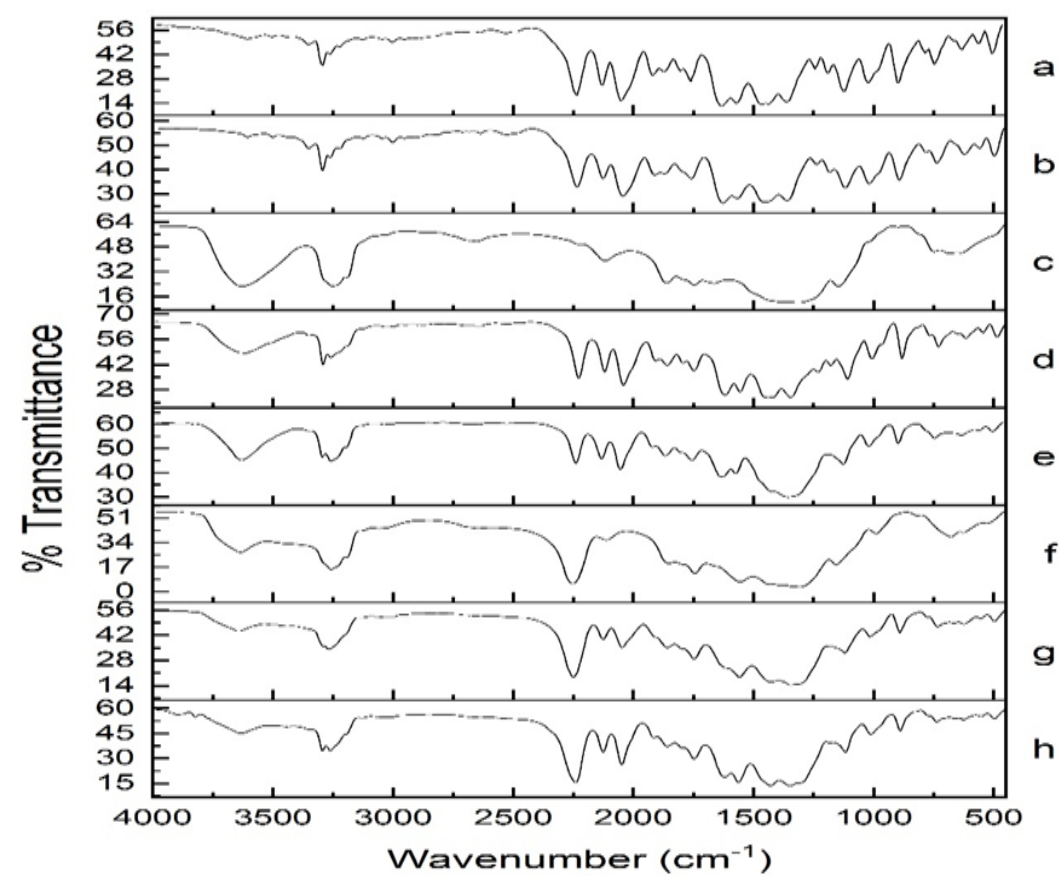

Fig. 3: FT-IR spectrographs of (a) FB (b) Spray dried FB (c) HPMC (d) HPMC physical mixture (e) HPMC solid dispersion (f) HPMCAS (g) HPMCAS Physical Mixture (h) HPMCAS solid dispersion

\section{DSC analysis}

Melting endotherms of FB and spray dried FB were observed at around $81.56{ }^{\circ} \mathrm{C}$ and $82.24^{\circ} \mathrm{C}$, respectively, confirming its crystalline state with no major effect of spray drying on endotherm shifting. Glass transition temperature of HPMC and HPMCAS were observed at around $170{ }^{\circ} \mathrm{C}$ and $120^{\circ} \mathrm{C}$, respectively. The physical mixtures of FB with different polymers, with the melting endotherms at $82.23{ }^{\circ} \mathrm{C}$ and $81.63^{\circ} \mathrm{C}$, confirmed the residual crystallinity due to the homogenous mixture formed (fig. 4). Furthermore, broad endothermic peaks in these mixtures could be attributed to the absence of endothermic relaxation [13]. Solid dispersions presented with no incidence of crystallinity, indicating perfect miscibility of drug and polymer to form stable solid dispersion for oral delivery [14].

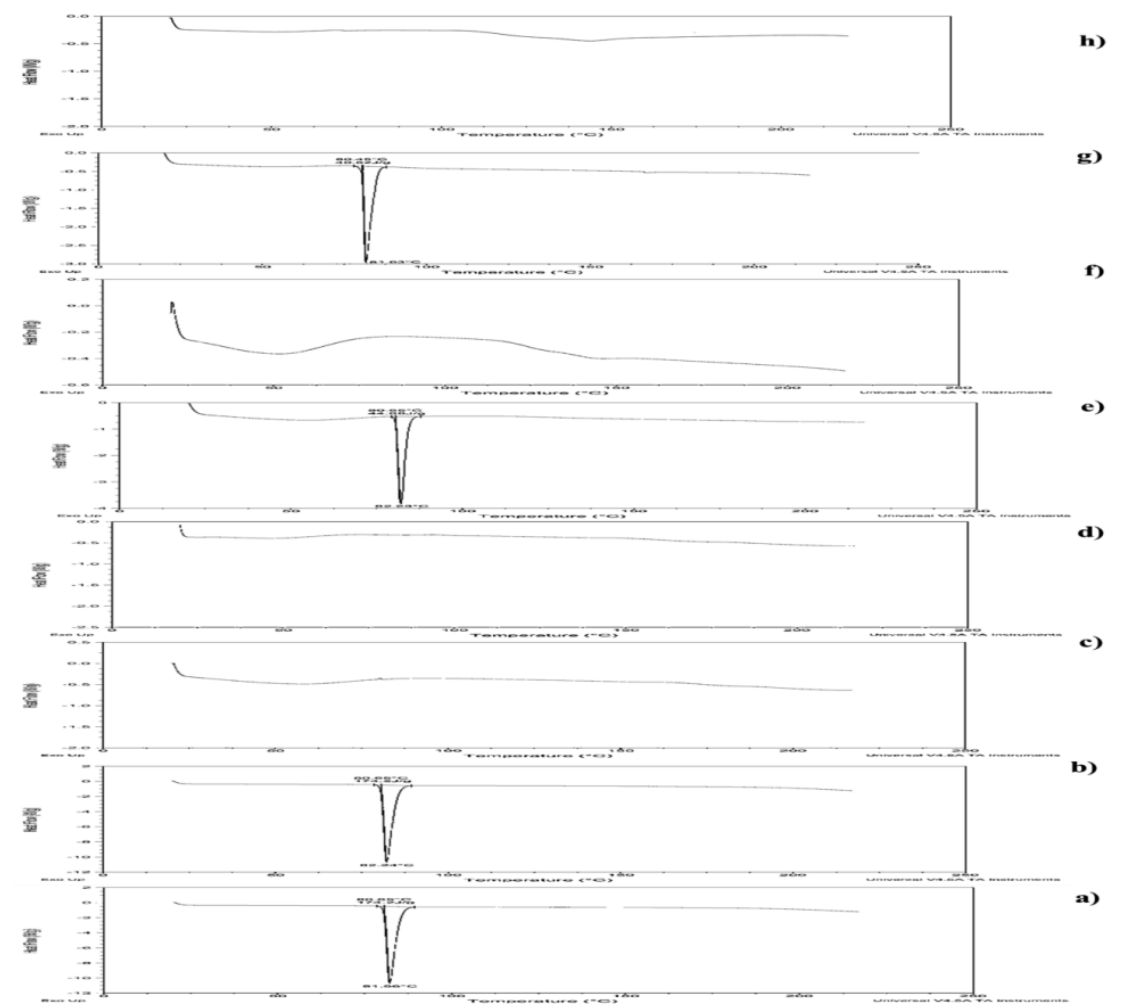

Fig. 4: DSC thermograms of (a) FB (b) Spray-dried FB (c) HPMC (d) HPMC physical mixture (e) HPMC solid dispersion (f) HPMCAS (g) HPMCAS Physical Mixture (h) HPMCAS solid dispersion 


\section{XRD analysis}

In the XRD of FB and spray-dried FB, sharp peaks were observed at a diffraction angle $(2 \theta)$ of $12.03^{\circ}, 14.49^{\circ}, 15.81^{\circ}, 16.31^{\circ}, 16.77^{\circ}$ $17.97^{\circ}, 19.37^{\circ}, 20.68^{\circ}, 20.94^{\circ}, 21.71^{\circ}, 21.89^{\circ}$ and $22.33^{\circ}$, indicating its crystalline nature and reduction of crystallinity was observed in spray-dried drug [15]. Partial amorphization was observed in the physical mixture, whereas, optimized solid dispersions observed with a reduction in the crystallinity index with an absence of diffraction patters indicating a change in overall geometry of crystalline to amorphous form and suggesting effective molecular dispersion of FB in solid dispersion formulation (fig. 5).

This phenomenon may be attributed to the attainment of molecular dispersion state in these formulations and confirmed the amorphous transformation of drug FB in the solid dispersions [15].

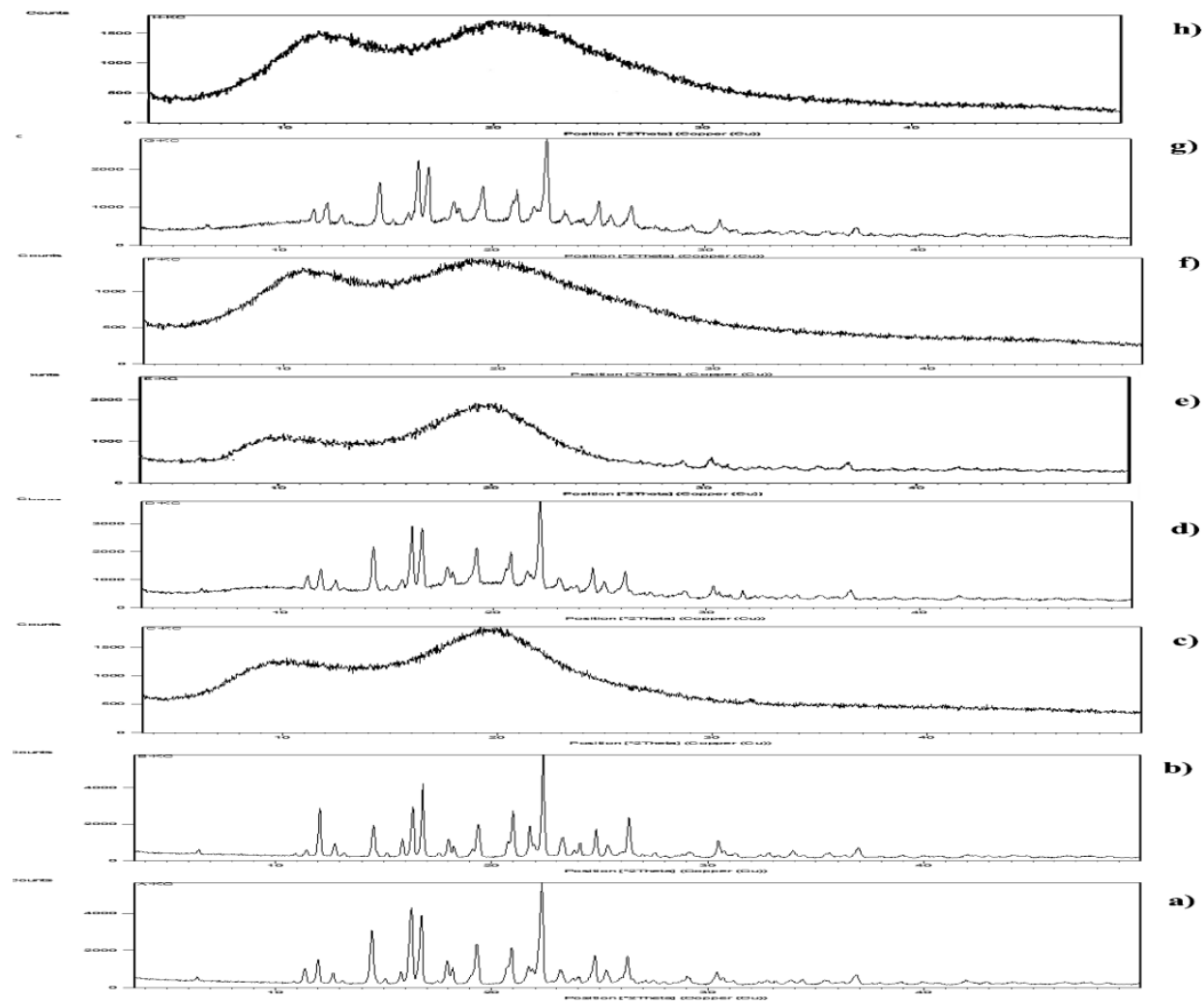

Fig. 5 (a): XRD patterns of (a) FB (b) spray-dried FB (c) HPMC (d) HPMC physical mixture (e) HPMC solid dispersion (f) HPMCAS (g) HPMCAS physical mixture (h) HPMCAS solid dispersion

\section{Scanning electron microscopy}

The particles of amorphous dispersions in SEM seemed to be collapsed and spherical in shape due to the rapid evaporation of solvent during spray drying (fig. 6). The size and morphology of spray dried amorphous solid dispersions confirms a similar surface state for solid dispersions of both the polymers, negotiating the effect of surface state on the dissolution rate [16].

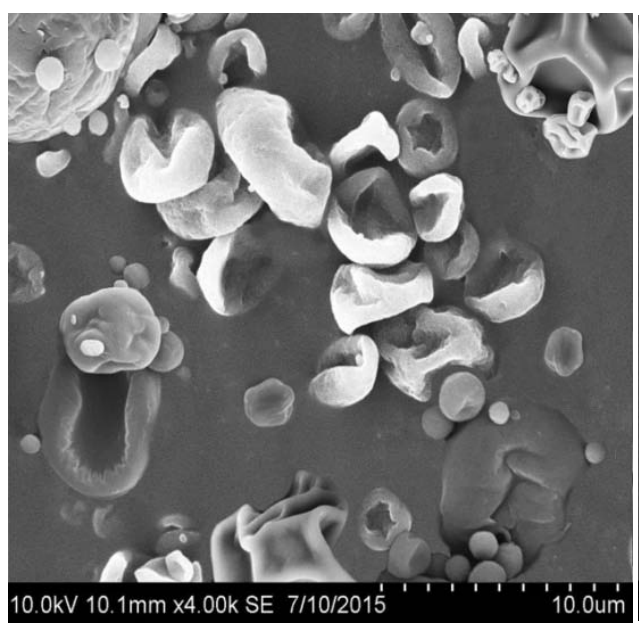

(a)

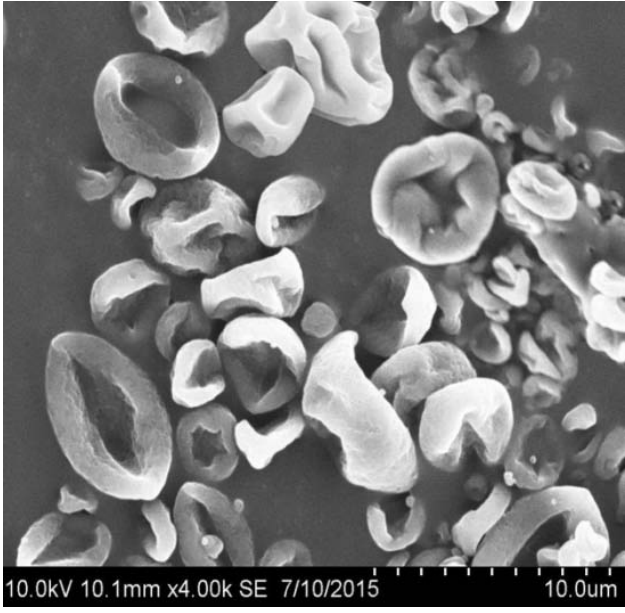

(b)

Fig. 6: SEM of (a) HPMC solid dispersion (b) HPMCAS solid dispersion 


\section{In vitro dissolution studies}

The in vitro release profile of FB, physical mixtures and optimized formulation in 0.05 M SLS is shown in fig. 7 and 8, respectively. A low release rate of $16.31 \%$ of pure drug indicated the need for enhancing the dissolution regime in order to develop a successful drug product. However, the presence of polymers significantly increased the dissolution of FB from the physical mixtures, i.e. PMC 1, PMC 2, PMC 3 containing HPMC and PM CAS 1, PM CAS 2 and PM CAS 3 containing HPMCAS. For HPMC, the rate of dissolution was found to be highest in the physical mixture PMC 3 i.e. $57.13 \%$ in $2 \mathrm{~h}$. In the case of HPMCAS, the percentage cumulative drug released (\%CDR) was highest in PM CAS 3 i.e. $62.84 \%$ in 2 h. This suggests that HPMCAS has a significant effect on the solubilisation of the drug as compared to HPMC in the formulation [16]. Moreover, it was observed that the dissolution of the drug from its carrier first increases and then it gets stable after some time, indicating the solubilizing effect of both the polymers $[17,18]$. This enhancement of dissolution of FB from HPMCAS can be described by several factors viz. lack of crystallinity, i.e., amorphization, increased wettability and dispersibility, and particle size reduction [19-21]. Also, prevention of aggregation during dissolution and particle size reduction could also be attributed to a better dissolution profile Overall, HPMCAS showed better augmentation in dissolution rate than HPMC and showed enhanced release rate than pure drug and marketed product.

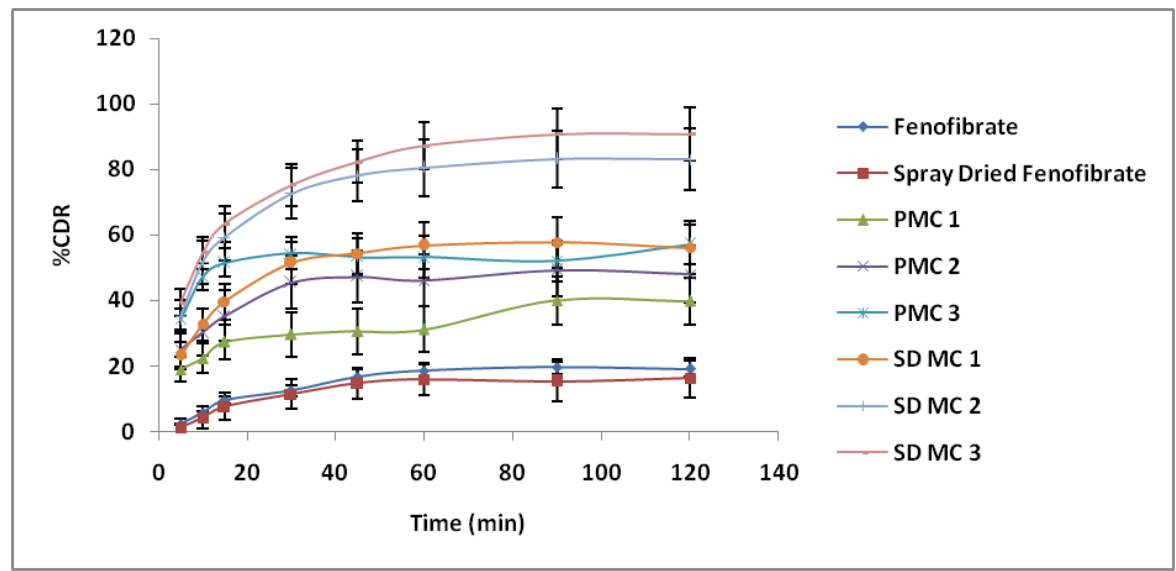

Fig. 7: Dissolution curves of FB, spray dried FB, physical mixtures and prepared solid dispersions of FB with HPMC (mean \pm SD, $n=3$ ) $(\mathrm{P}<0.05$, compared PMC 1-PMC 3 with pure Fb) $(\mathrm{P}<0.001$, compared SD MC 1-SD MC 3 with pure FB)

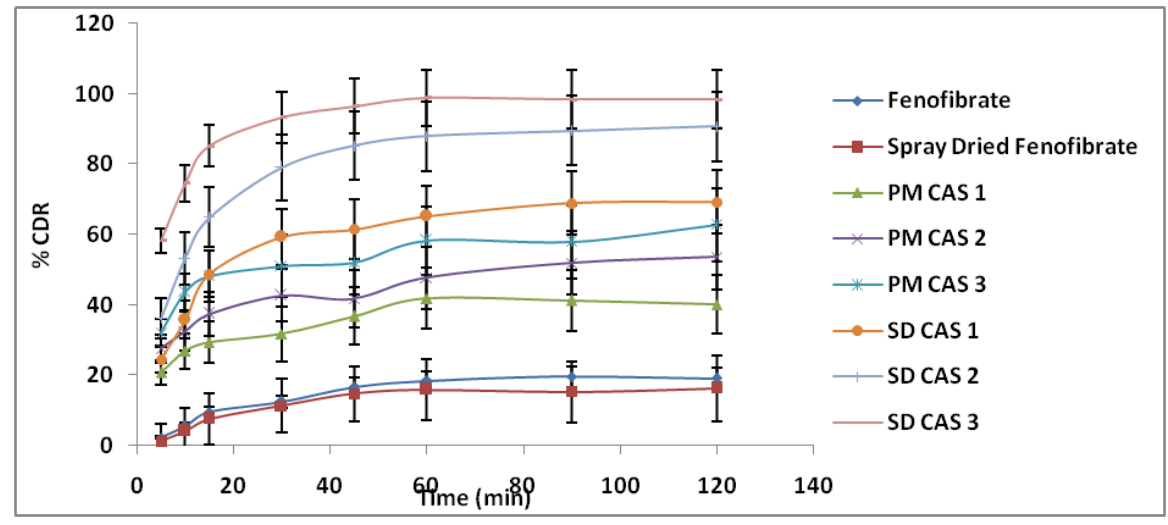

Fig. 8: Dissolution curves of FB, spray dried FB, physical mixtures and prepared solid dispersions prepared with HPMC and HPMCAS $(m e a n \pm S D, n=3)(P<0.05$, compared PM CAS1-PM CAS 3 with pure $F B)(P<0.001$, compared SD CAS 1-SD CAS 3 with pure FB)

\section{Dissolution parameters of solid dispersions of FB with HPMC and HPMCAS}

The values for various dissolution parameters calculated for six solid dispersions prepared by using the different drug to polymer ratios are shown in table 2. It was evident that HPMCAS enhanced the dissolution rate of the drug to a greater extent. HPMCAS dissolution curve shoots up quickly than HPMC dissolution curve and was better at initiation and maintenance of supersaturation. It can be seen that solid dispersions SD3 and SD6 showed best results i.e. have the highest values for $\mathrm{DE}_{60}$ for HPMC and HPMCAS, respectively [22].

Table 2: Dissolution parameters of solid dispersions

\begin{tabular}{llllll}
\hline Solid dispersion batches & $\mathbf{D E}_{\mathbf{3 0}}$ & $\mathbf{D E}_{\mathbf{6 0}}$ & $\mathbf{D P}_{\mathbf{5}}$ & $\mathbf{D P}_{\mathbf{3 0}}$ & $\mathbf{D P}_{\mathbf{1 2 0}}$ \\
\hline SD MC 1 & $39.40 \%$ & $44.83 \%$ & $23.26 \%$ & $51.48 \%$ & $56.14 \%$ \\
SD MC 2 & $52.41 \%$ & $64.90 \%$ & $35.21 \%$ & $72.65 \%$ & $82.94 \%$ \\
SD MC 3 & $55.54 \%$ & $68.66 \%$ & $39.23 \%$ & $75.29 \%$ & $90.82 \%$ \\
SD CAS 1 & $41.14 \%$ & $51.49 \%$ & $24.52 \%$ & $59.27 \%$ & $69.24 \%$ \\
SD CAS 2 & $56.32 \%$ & $70.33 \%$ & $36.12 \%$ & $79.09 \%$ & $90.72 \%$ \\
SD CAS 3 & $73.81 \%$ & $85.01 \%$ & $58.26 \%$ & $93.23 \%$ & $98.37 \%$ \\
\hline
\end{tabular}




\section{CONCLUSION}

We focused on the comparison of HPMC and HPMCAS as the versatile hydrophilic polymers for the attainment of amorphization and enhancement of in vitro dissolution. The formed solid dispersion was in the amorphized state in the solid state characterizations and revealed highest in the release, particularly with HPMCAS based solid dispersion. Multi-folds augmentation in release behavior of optimized solid dispersions prepared with HPMCAS was observed and was statistically significant $(p<0.05)$ as compared with the pure API. These studies revealed that even though the drug remains in amorphous form in solid dispersions based on both the polymers, HPMCAS based amorphous solid dispersions demonstrated improved dissolution profile as compared to HPMC based dispersions.

\section{ACKNOWLEDGEMENTS}

The authors are highly thankful to Macleod's Pharmaceuticals, Mumbai for providing gift sample of fenofibrate. Emerging Life Sciences Facility in Guru Nanak Dev University for carrying out characterization studies is highly acknowledged.

\section{AUTHORS CONTRIBUTIONS}

All the authors have contributed equally

\section{CONFLICT OF INTERESTS}

Declared none

\section{REFERENCES}

1. Amidon GL, Lennernas H, Shah VP, Crison JR. A theoretical basis for a biopharmaceutic drug classification: the correlation of in vitro drug product dissolution and in vivo bioavailability. Pharm Res 1995;12:413-20.

2. Pamudji JS, Wikarsa S, Tampara MH. Improvement of gliclazide's dissolution rate by using surface solid dispersion with avicel ph 101. Int J Pharm Pharm Sci 2014;6:461-5.

3. Mohanty S, Pal A. Dissolution enhancement of seroquel by solid dispersion. Asian J Pharm Clin Res 2016;9:284-7.

4. Nikghalb LA, Singh G, Singh G, Kahkeshan KF. Solid dispersion: methods and polymers to increase the solubility of poorly soluble drugs. J Appl Pharm Sci 2012;2:170-5.

5. Paudel A, Worku ZA, Meeus J, Guns S, Van den Mooter G. Manufacturing of solid dispersions of poorly water-soluble drugs by spray drying: formulation and process considerations. Int J Pharm 2013;453:253-84.

6. Dixit M, Rasheed A, Fijaz Rahman NC, Daniel S. Enhancing solubility and dissolution of fenofibrate by spray drying technique. Int J Pharm Pharm Sci 2015;7:173-7.

7. Friesen DT, Shanker R, Crew M, Smithey DT, Curatolo WJ, Nightingale JA. Hydroxypropyl methylcellulose acetate succinate-based spray-dried dispersions: an overview. Mol Pharm 2008;5:1003-19.

8. Tanno F, Nishiyama Y, Kokubo H, Obara S. Evaluation of hypromellose acetate succinate (HPMCAS) as a carrier in solid dispersions. Drug Dev Ind Pharm 2004;30:9-17.

9. Jamzad S, Fassihi R. Role of surfactant and $\mathrm{pH}$ on dissolution properties of fenofibrate and glipizide-a technical note. AAPS PharmSciTech 2006;7:E1-E6.

10. Khan KA, Rhodes CT. Effect of compaction pressure on the dissolution efficiency of some direct compression systems. Pharm Acta Helv 1972;47:594-607.

11. Costa P, Sousa Lobo JM. Modeling and comparison of dissolution profiles. Eur J Pharm Sci 2001;13:123-33.

12. Xie Y, Li G, Yuan Y, Cai Z, Rong R. Preparation and in vitro evaluation of solid dispersions of total flavones of Hippophae rhamnoides L. AAPS PharmSciTech 2012;10:631-40.

13. Mura P, Faucci MT, Manderioli A, Bramanti G, Parrini P. Thermal behavior and dissolution properties of naproxen from binary and ternary solid dispersions. Drug Dev Ind Pharm 1999;25:257-64.

14. Ghosh I, Snyder J, Vippagunta R, Alvine M, Vakil R, Tong WQ, et al. Comparison of HPMC based polymers performance as carriers for manufacture of solid dispersions using the melt extruder. Int J Pharm 2011;419:12-9.

15. Curatolo W, Nightingale JA, Herbig SM. Utility of hydroxypropylmethylcellulose acetate succinate (HPMCAS) for initiation and maintenance of drug supersaturation in the GI milieu. Pharm Res 2009;26:1419-31.

16. Betageri GV, Makarla KR. Enhancement of dissolution of glyburide by solid dispersion and lyophilization techniques. Int J Pharm 1995;126:155-60.

17. Matsuda Y, Otsuka M, Onoe M, Tatsumi E. Amorphism and physicochemical stability of spray-dried furosemide. J Pharm Pharmacol 1992;44:627-33.

18. Nandiyanto ABD, Okuyama K. Progress in developing spraydrying methods for the production of controlled morphology particles: from the nanometer to submicrometer size ranges. Adv Powder Technol 2011;22:1-19.

19. Shah TJ, Amin AF, Parikh JR, Parikh RH. Process optimization and characterization of poloxamer solid dispersions of a poorly water-soluble drug. AAPS PharmSciTech 2006;8:18-24.

20. Tanno F, Nishiyama Y, Kokubo H, Obara S. Evaluation of hypromellose acetate succinate (HPMCAS) as a carrier in solid dispersions. Drug Dev Ind Pharm 2004;30:9-17.

21. Tashtoush BM, Al-Qashi ZS, Najib NM. In vitro and in vivo evaluation of glibenclamide in solid dispersion systems. Drug Dev Ind Pharm 2004;30:601-17.

22. Surikutchi BT, Patil SP, Shete G, Patel S, Bansal AK. Drugexcipient behavior in polymeric amorphous solid dispersions. J Excipients Food Chem 2013;4:70-94. 